\title{
Aplicaciones Didácticas de las TIC's a la enseñanza de la Geografía en Secundaria: El estudio de la población española a través del paisaje
}

\author{
Arantxa García Vidales. Universidad de Valladolid \\ José Luis García Cuesta. Universidad de Valladolid \\ Recepción: 15 de enero de 2016 | Aceptado: 16 de enero de 2017 \\ Correspondencia: José Luis García Cuesta | Correo-e: josgar@fyl.uva.es
}

\begin{abstract}
(iD) 0000-0002-9217-9436
Citar: García Vidales, A. y García Cuesta, J.L. (2017). Aplicaciones Didácticas de las TIC's a la enseñanza de la Geografía en Secundaria: El estudio de la población española a través del paisaje. ReiDoCrea, 6, 37-49.
\end{abstract}

\begin{abstract}
Resumen: Las Tecnologías de la Información y la Comunicación, TICs, han ido alcanzando -sobre todo en las dos últimas décadas- un gran desarrollo e importancia en nuestra sociedad, conformando un conjunto de herramientas empleadas frecuentemente en muy distintos ámbitos de nuestra vida, incluido el educativo (Alcántara Trapero, 2009). Este trabajo analiza la importancia, valor y utilidad que poseen las TICs en la enseñanza de la Geografía, no solo como una mera herramienta para la visualización de contenidos, sino como un conjunto de recursos con los que poder desarrollar otros métodos y formas de enseñanza-aprendizaje, más eficaces y atractivos en la Educación Secundaria. También se aborda un análisis y repaso documental sobre el tema, partiendo de la lectura y revisión de la legislación existente al respecto, del estudio de los recursos formativos y pedagógicos existentes, y de la utilización de métodos de análisis cualitativo y cuantitativo. Del mismo modo, se presenta, a modo de ejemplo, una propuesta metodológica para la enseñanza de la Geografía, empleando los denominados "Story Map", a partir de una herramienta ofrecida por ArcGIS Online que permite vincular documentos e información de muy diversas procedencias y formatos, asociándolos a la realidad territorial del ámbito de estudio. Como resultados, se ha apreciado la importancia que posee integrar correctamente las TIC's en nuestras vidas $\mathrm{y}$, concretamente, dentro del ámbito educativo, ya que éstas no solo consiguen mejorar el estudio y comprensión de las materias en el aula, sino que, al integrarse en los procesos de enseñanzaaprendizaje, logran que la asimilación de contenidos por parte de los alumnos sea mucho más activo, ya que el alumno adopta un nuevo "rol" en su propio aprendizaje, siendo un agente que colabora y participa en la elaboración de sus conocimientos.
\end{abstract}

Palabras clave: Enseñanza de la Geografía | Innovación Educativa

Didactic ICT Applications for Teaching Geography in Secondary: The Study of the Spanish Population through the Landscape

\begin{abstract}
Information and communications technologies (henceforward ICTs) have developed considerably and attained great importance in our society, especially over the last two decades. They make up a set of tools used frequently in very different spheres of our lives, including the educational sphere (Alcántara Trapero, 2009). This article aims to analyse the importance, value and utility of the ICTs in the teaching of Geography, not merely as a tool to visualize content, but also as a set of resources with which to develop other more effective and attractive methods and ways of teaching-learning for secondary education. We carry out a documentary analysis and review of the subject, starting from a reading and review of the existing legislation of the topic, a study of the existing pedagogical and instructional resources, and the use of qualitative and quantitative analysis methods. Similarly, by way of example, we present a methodological proposal for the teaching of Geography, using the so-called "Story Map", based on a tool offered by ArcGIS Online which allows documents and information from many different sources and in many different formats to be linked together, associating them with the territorial reality of the sphere of study. The results of our study have shown us the importance of correctly integrating the ICTs into our lives and, more specifically, within the educational sphere, since they not only improve the study and comprehension of the subjects in the classroom, but also, when they are integrated in the teachinglearning process, the result is a much more active assimilation of the content on the part of the students. This is because the students adopt a new "role" in their own learning; they become agent who participate and collaborate in the development of their knowledge base.
\end{abstract}

Key words: Geography Education | Educational Innovation 


\section{Introducción}

La enseñanza en la Geografía, como en el resto de las materias educativas, ha ido avanzando a lo largo del tiempo, es decir, las formas de enseñar y aprender no han sido siempre las mismas, ya que éstas se han ido adaptando no solo a los intereses y necesidades de cada momento, sino también a los recursos y medios disponibles para ello (Kerski, 2011). Así, las nuevas tecnologías, y más concretamente las TIC's, han ido alcanzando progresivamente un papel cada vez más importante -e incluso imprescindible- en el día a día de las aulas y en los actuales procesos de enseñanzaaprendizaje. Esta importancia se refleja claramente en los textos de varias leyes y decretos de nuestro país, tanto en legislación anterior como en la actual ley educativa que rige los planes de estudio, la LOMCE, señalando en varios de sus artículos a las TIC como elementos necesarios para el crecimiento y desarrollo, tanto profesional como personal, de los estudiantes del siglo XXI. A modo de ejemplo, en el Art. 111 bis, punto 5, se recoge la importancia de promover el uso de las TIC, por parte de las Administraciones educativas y los equipos directivos de los distintos centros, como "un medio didáctico apropiado y valioso para llevar a cabo las tareas de enseñanza y aprendizaje", y en el punto 6 del mismo artículo se señala la necesidad de la formación permanente de los docentes en la competencia digital, logrando facilitar así el desarrollo de la cultura digital en el aula. De hecho, dentro de las distintas competencias a desarrollar (Ver Tabla 1.), se hace referencia a la denominada "Competencia digital", lo que da muestra de la importancia que poco a poco se le ha ido otorgando a las TIC's en el marco legislativo de Educación.

\begin{tabular}{|c|c|}
\hline \multicolumn{1}{|c|}{ Tabla 1.- Competencias actuales en el currículo de secundaria } \\
\hline COMPETENCIAS EN EDUCACIÓN SECUNDARIA \\
\hline - & Competencia en comunicación lingüística \\
- & Competencia matemática \\
- Competencia en el conocimiento y la interacción con el mundo físico, tratamiento de la información y \\
- Competencia digital \\
- Competencia social y ciudadana \\
\hline Elaborado a partir de Arrebola Haro, J.C. (2014).
\end{tabular}

Por otro lado, el desarrollo de esta "competencia digital" debe acompañarse, tal y como sostienen Buzo Sánchez (2014) y Pico (2013), de una modificación y/o adaptación de los métodos y formas de enseñanza, de tal forma que las TIC lleguen a conformar una parte -quizá incluso la más importante- en el proceso de aprendizaje de los alumnos de enseñanza secundaria. Del mismo modo, y siguiendo los planteamientos de Sánchez Asín (2009) y otros muchos autores relacionados con la materia, las TIC suponen un nuevo recurso educativo o forma de trabajo de carácter innovador realmente interesante y útil en la actualidad, puesto que ayudan y facilitan tanto los procesos de aprendizaje en las aulas, como el autoaprendizaje o aprendizaje autónomo por parte de los alumnos dentro y fuera de ellas. Por ello, es necesario adaptar y aunar los métodos y técnicas tradicionales que posean interés -en el caso de la Geografía, por ejemplo, las salidas de trabajo campo- con las herramientas, recursos y metodologías más innovadoras (proyectores, PDI, PPT, ...). Del mismo modo, este nuevo enfoque exige en cierto modo una nueva actitud -nuevos roles- por parte de los distintos agentes intervinientes en el proceso educativo, principalmente en los docentes, alumnos y los propios centros educativos (Oller y Villanueva, 2007; Muñoz Carril y González Sanmamed, 2013). En este sentido, se hace necesario invertir tanto en la mejora y adaptación de los equipamientos e infraestructuras existentes, como en modificar ciertas prácticas "tradicionales" llevadas a cabo en el aula. En el caso concreto de la Geografía, y tal como Herrera Ávila (2012) y Delgado 
Mahecha (1989) señalan, buena parte de las clases siguen caracterizándose por un relato descriptivo y enumerativo de un sinfín de conceptos y temas, unos tras otros, siguiendo el orden establecido en el temario, pero sin apenas relación entre ellos ignorando el componente integrador que tiene el territorio y, además, con una baja 0 ausente participación del alumno en el desarrollo de las clases. Esto genera, no solo una falta de atractivo e interés por parte del alumno en aquello que se le enseña, sino también un déficit en el desarrollo de competencias, es decir, de las capacidades y habilidades adquiridas por el estudiante que le permitan aplicar conocimientos, tanto en su futura vida profesional como en la cotidiana. Por tanto, las TIC's deben generar y venir acompañadas en su integración en las aulas por un cambio en la forma de organizar los procesos de enseñanza-aprendizaje, de tal forma que su función sea la de contribuir a un aprendizaje mucho más eficiente, consolidado y activo por parte de los alumnos, tanto dentro como fuera del aula (Gómez Trigueros,2010; Sánchez Asín et al., 2009).

Para todo ello, la figura del docente adquiere un papel fundamental ya que es el principal encargado de emplear dichos recursos en el aula, saber qué herramientas son necesarias en cada momento -selección de materiales- y dotarlas de contenidos que contribuyan al aprendizaje y la adquisición de conocimientos y competencias por parte de los estudiantes, es decir, que su función sea la de "enseñar a aprender a lo largo de toda la vida,..., adaptarse a una sociedad en constante evolución" (MartínLaborda, 2005); para lo cual la formación continua y permanente por parte del profesorado adquiere un significado y transcendencia muy significativa (Tello Díaz y Aguaded Gómez, 2009; Almerich et al., 2010).

\section{Material y métodos}

A partir de la información recopilada por medio del estudio y análisis pormenorizado de numerosos textos referentes al tema que nos ocupa, así como de lo que exige la actual ley educativa, se hace necesario avanzar en los métodos de enseñanzaaprendizaje basados en el "e-learning" y el uso de las TIC's como base fundamental para el aprendizaje y adquisición de competencias por parte de los estudiantes de educación secundaria, ya que éstas herramientas suponen un nuevo "método o estrategia didáctica" (Alcántara Trapero, 2009), una nueva forma de trabajo adaptada a las necesidades y exigencias de la sociedad actual, con la que además se fomentan las relaciones entre los distintos agentes intervinientes en el proceso educativo, mejorándolo así en su conjunto.

Por todo ello, planteamos una propuesta metodológica que trata de adaptarse y atender a esas necesidades formativas y competenciales, exigidas a los actuales estudiantes de educación secundaria. Con ella, además, se pretende adaptar, combinar e integrar los contenidos establecidos en el currículo de la actual ley educativa con una forma diferente de abordar y enseñar el temario que se recoge en dicha legislación. Al mismo tiempo, y como ya hemos mencionado, nos centramos en el caso concreto del estudio de la Geografía en la educación secundaria, por lo que pretendemos que, a través del estudio de la Geografía, los alumnos consigan entender e interpretar el territorio, apoyándose para ello en el relato que nos ofrecen los paisajes. En este sentido, tratar de entender los paisajes obliga y exige, en cierto modo, comprender las relaciones y significados que posee el medio, es decir, saber analizar la acción del hombre sobre éste en función de las necesidades e intereses existentes en cada momento, interpretar las distintas estructuras y organización de los espacios, en los que no solo interviene la propia labor de la naturaleza, sino también la mano del hombre (Arrebola Haro, 2014; Bertrand, 2010). Por tanto, como afirma Bertrand (2008), entendemos el paisaje como el resultado, la representación de la 
realidad territorial conformada por los distintos elementos (materiales e inmateriales) que lo constituyen, como consecuencia de las diversas actividades y acciones encaminadas a la explotación y aprovechamiento del territorio a lo largo del tiempo, por lo que, el paisaje es el que en definitiva dota de un significado y sentido a los distintos espacios a lo largo de la historia y de las sociedades que se han sucedido en él (Rodríguez Eguizábal, 1991). (Ver Figura 1)

Así, otro de los objetivos que hemos perseguido con la elaboración de esta propuesta metodológica es que los alumnos aprendan, a través del estudio del paisaje, a desgranar e interpretar las distintas "capas" de las que se compone el territorio, de tal forma que el estudio de un determinado tema se enlace y relacione con otros. Igualmente, la propuesta de "Story Map" que aquí se presenta pretende ser un recurso útil y válido tanto para las explicaciones desarrolladas en el aula por parte del profesor, como también una herramienta de apoyo para el aprendizaje y construcción de conocimiento autónomo del alumno, es decir, que contribuya a la maduración personal y profesional y a la adquisición de competencias que venimos mencionando. Sin embargo, y dado que el desarrollo completo de todos los elementos conformadores de un paisaje y tipos de paisaje existentes es realmente extenso, nos hemos centrado en un apartado concreto, el de la población española, puesto que además de que el hombre es el principal elemento o agente integrador y generador de paisajes, suele ser uno de los temas tratados en las PAU.

Por tanto, para la elaboración de la propuesta metodológica hemos analizado y nos hemos apoyado en varios aspectos: en primer lugar, qué exigencias impone la actual ley educativa para la educación secundaria de la Geografía y, más concretamente, para el curso de $2^{\circ}$ de bachillerato (contenidos, competencias,...); en segundo lugar, hemos consultado el Anexo IV -relativo a los contenidos exigibles- que actualmente establece la región de Castilla y León en la PAU, deteniéndonos en lo especificado en el tema 17, puesto que es el que trata sobre la población española en la cual centraremos el desarrollo del Story Map; y, por último, ayudándonos de varios libros de texto de Geografía para $2^{\circ}$ de bachillerato, hemos ido elaborando y maquetando en el Story Map los contenidos referentes al tema de la población española, apoyando su enseñanza y aprendizaje en el estudio de los paisajes, ya que ello nos permite poder tratar otros aspectos que forman parte del territorio, lo que en definitiva implica estudiar la Geografía pero, en el caso concreto que aquí proponemos, a través de una "nueva mirada", de una interpretación del territorio empleando las TIC, una forma de "smart learning" que favorezca un aprendizaje más crítico y reflexivo por parte de los estudiantes, ya que es lo que realmente les dotará de las competencias necesarias para afrontar los retos y satisfacer las necesidades e intereses exigidos por la sociedad del siglo XXI (Gil Durán et al., 2014). 


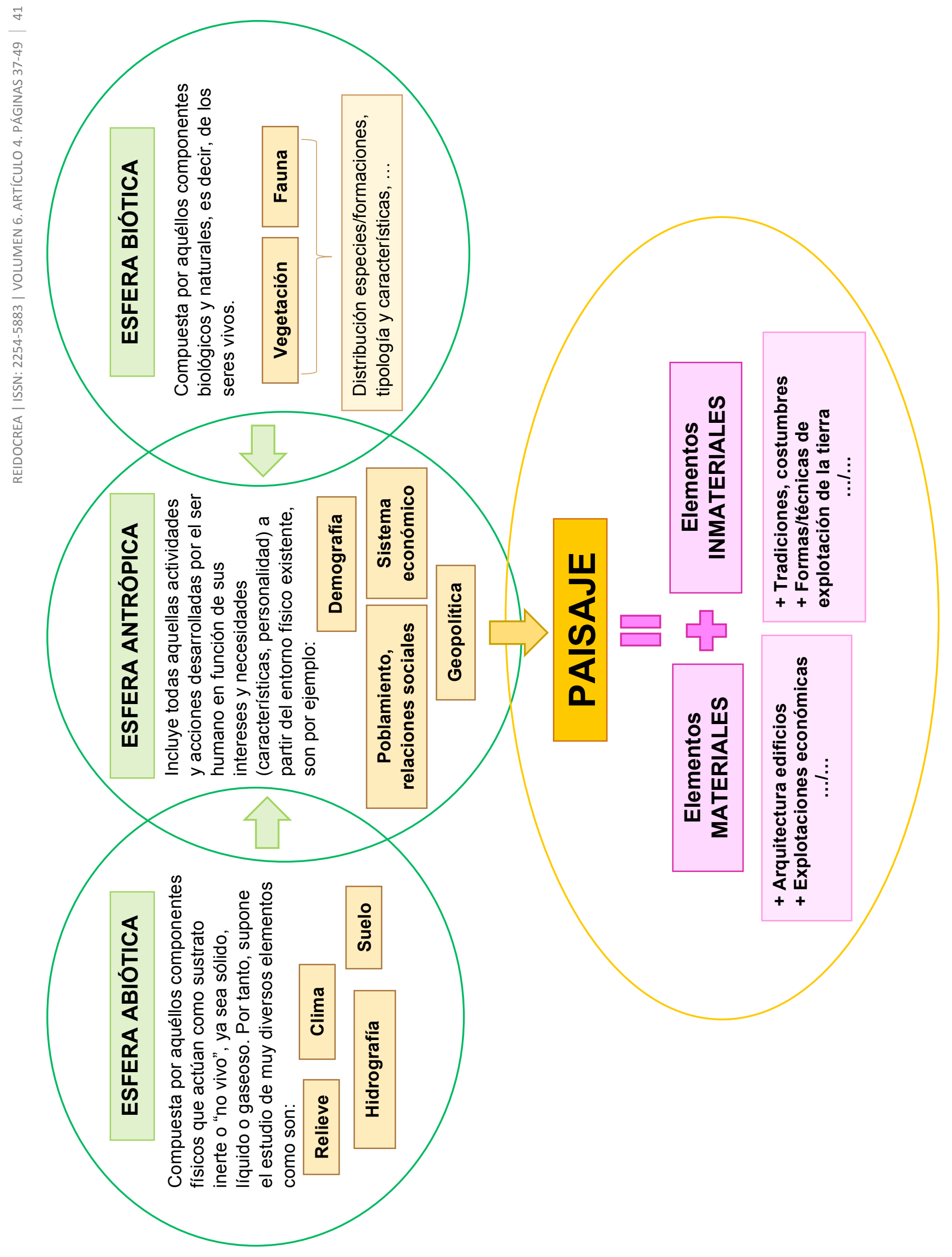


Como bien hemos señalado, para la elaboración de la propuesta metodológica hemos empleado la herramienta "Story Map" ofrecida por la plataforma ArcGIS-Online de ESRI, un sistema SIG apoyado en la web de colaboración on-line, es decir, una plataforma abierta y basada en la nube de Internet que ofrece la posibilidad de crear, usar y compartir diverso contenido de carácter geográfico y/o geoespacial. Además, mediante el uso de esta plataforma, todos los recursos e información generada, pueden estar disponibles y ser accesibles para todos los miembros de una organización concreta, o al público en general, así como que el contenido pueda estar disponible en cualquier momento y a través de muy diversos dispositivos, algo realmente interesante ya que hoy en día la mayor parte de la sociedad $-y$, fundamentalmente, los estudiantes- utilizan sus dispositivos móviles o tablets a diario, por lo que poder acceder a información desde dichos dispositivos facilita enormemente que éstos se impliquen y sientan un mayor interés de cara a su formación. Al mismo tiempo, con esta propuesta metodológica se pretende que los docentes conozcan un nuevo recurso TIC con el que poder diseñar y elaborar sus propios materiales didácticos, a la par que pueden lograr que sean más atractivos, flexibles y útiles para su aprendizaje por los alumnos. Otro motivo por el que hemos elegido los Story Map, es porque permiten trabajar y publicar contenidos en la web sin necesidad de disponer de conocimientos previos de programación, o del manejo de procesos informáticos complejos. Además, los Story Map emplean la técnica conocida como "storytelling", que consiste en desarrollar procesos de enseñanza-aprendizaje basados y apoyados en la narración de historias en las que la información aparece representada, y puede ser visualizada a través de distintos recursos y elementos (mapas, vídeos, imágenes, gráficos...) (De Lázaro y Torres et al., 2016; González González y Pérez Pastrana, 2014). Dentro de la gran variedad de plantillas o modelos ofrecidos en la propia plataforma, hemos elegido como story map principal el modelo "Map Series", que nos permite ordenar los contenidos en una serie de pestañas. De ésta forma, la información de los distintos puntos o apartados incluida en nuestra propuesta metodológica se ha dispuesto -en general- siguiendo el esquema de la Figura 2.

Figura 2.- Disposición general de la información en la aplicación web (Story Map)

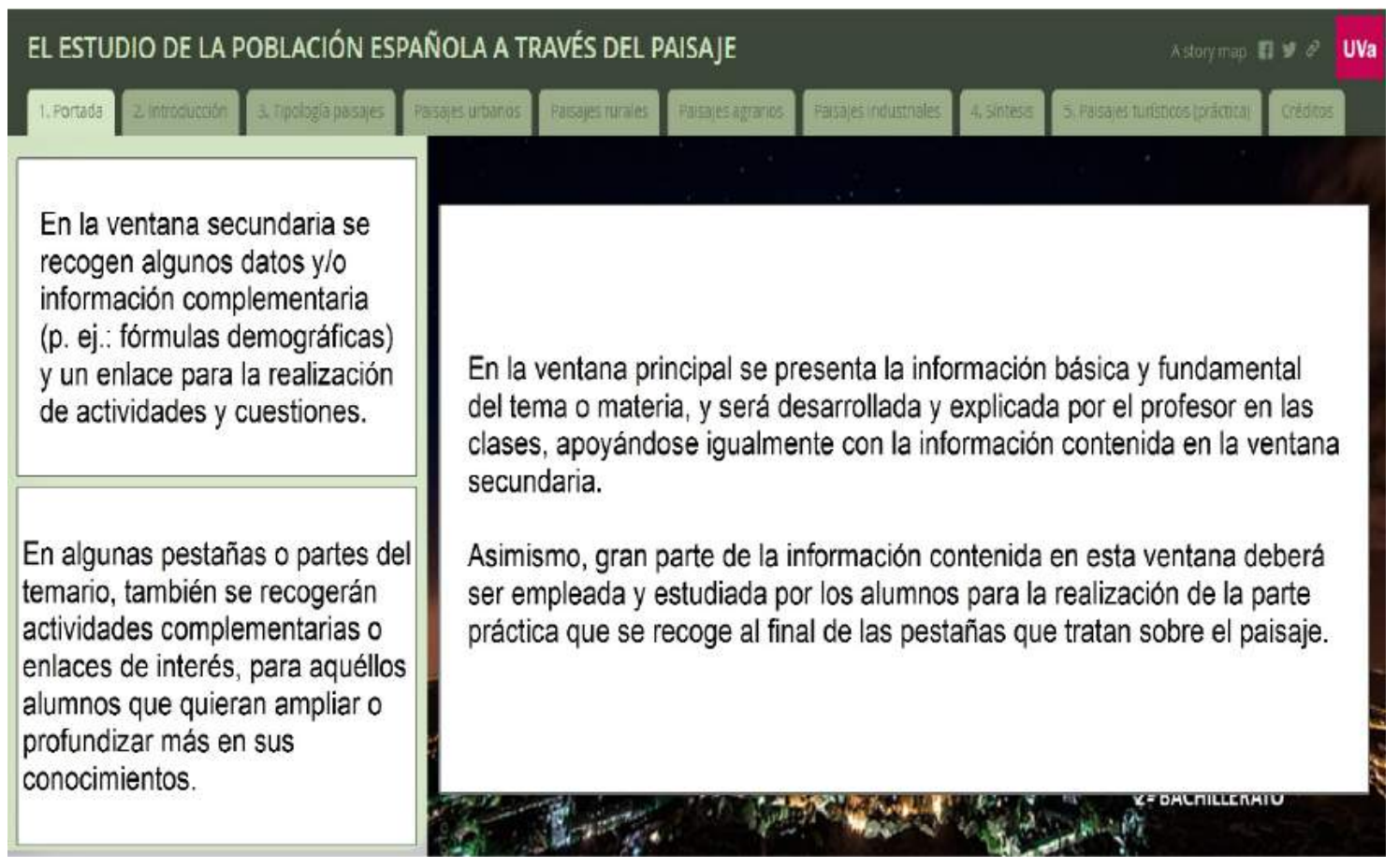


Asimismo, para la elaboración de la aplicación web, hemos combinado recursos elaborados por nosotros mismos con otros tomados de varios autores, dadas las limitaciones y restricciones -fundamentalmente a la hora de subir "shapes" de un determinado tamaño- que posee la cuenta como usuario gratuito (cuenta pública) en ArcGIS Online. Finalmente, y de manera simultánea a la maquetación de la información y los contenidos en el Story Map, elaboramos un cuadro-resumen a modo de guía y síntesis con los principales objetivos y los contenidos existentes en cada uno de los apartados de la aplicación web.

\begin{tabular}{|c|c|c|}
\hline $\begin{array}{l}\text { Apartados del } \\
\text { Story Map }\end{array}$ & Principal/es objetivo/s & Contenidos \\
\hline $\begin{array}{l}\text { Introducción y } \\
\text { tipología } \\
\text { paisajes }\end{array}$ & $\begin{array}{l}\text { Ofrecer una pequeña explicación sobre qué } \\
\text { entendemos por paisaje en la actualidad y } \\
\text { de qué elementos se compone, de tal forma } \\
\text { que sirva como soporte teórico inicial sobre } \\
\text { el cual poder profundizar y desarrollar otras } \\
\text { ideas }\end{array}$ & $\begin{array}{l}\text { Definición de paisaje, aspectos a tener en cuenta } \\
\text { según el concepto actual, principales elementos o } \\
\text { agentes conformadores, referencia a la diversidad } \\
\text { de paisajes geográficos }\end{array}$ \\
\hline $\begin{array}{l}\text { Paisajes } \\
\text { urbanos }\end{array}$ & $\begin{array}{l}\text { Desarrollar los contenidos sobre la } \\
\text { evolución y distribución de la población en } \\
\text { España y la repercusión o incidencia de } \\
\text { todo ello en los paisajes urbanos; asimismo, } \\
\text { se pretende enseñar el manejo de las } \\
\text { principales fuentes demográficas }\end{array}$ & $\begin{array}{l}\text { Distribución espacial de la población (densidad en } \\
\text { España), evolución población española, } \\
\text { transformación y cambios en los paisajes de las } \\
\text { áreas urbanas, importancia sector servicios }\end{array}$ \\
\hline Paisajes rurales & $\begin{array}{l}\text { Tratar el tema de la dinámica natural y el } \\
\text { crecimiento demográfico en España, } \\
\text { mostrando igualmente la actual situación de } \\
\text { dicho crecimiento en las áreas rurales y los } \\
\text { paisajes resultantes de todo ello }\end{array}$ & $\begin{array}{l}\text { Tipos de poblamiento, situación actual de los } \\
\text { componentes de crecimiento natural esenciales, } \\
\text { transición demográfica España, desigualdades } \\
\text { espaciales en la dinámica interna, factores } \\
\text { estructurales fundamentales (envejecimiento, ...), } \\
\text { sector primario }\end{array}$ \\
\hline $\begin{array}{l}\text { Paisajes } \\
\text { agrarios }\end{array}$ & $\begin{array}{l}\text { Desarrollar los contenidos relativos a las } \\
\text { migraciones interiores y exteriores en } \\
\text { España y la incidencia de todo ello en los } \\
\text { paisajes, y mostrar algunos paisajes } \\
\text { agrarios del país }\end{array}$ & $\begin{array}{l}\text { Distribución espacial y evolución de la población } \\
\text { inmigrante, factores y causas de dicha situación, } \\
\text { modelos migratorios, consecuencias demográficas } \\
\text { (edad media, población activa, pirámide población, } \\
\text {...), los nuevos paisajes agrícolas, relación con } \\
\text { otras variables en el territorio (ej: medio físico) }\end{array}$ \\
\hline $\begin{array}{l}\text { Paisajes } \\
\text { industriales }\end{array}$ & $\begin{array}{l}\text { Estudiar el tema de la estructura económica } \\
\text { de la población en España }\end{array}$ & $\begin{array}{l}\text { Distribución espacial de la población en empleo } \\
\text { industrial, evolución importancia de la actividad, } \\
\text { paisajes resultantes (evolución, diferencias), tasas } \\
\text { paro y actividad, estructura sectorial }\end{array}$ \\
\hline Sintesis & Destacar las principales ideas & Transformaciones importantes en el paisaje \\
\hline $\begin{array}{l}\text { Paisajes } \\
\text { turísticos } \\
\text { (práctica) }\end{array}$ & $\begin{array}{l}\text { Analizar y entender qué transformaciones } \\
\text { territoriales y paisajísticas han tenido lugar } \\
\text { con la aparición del turismo }\end{array}$ & Web Map de elaboración conjunta \\
\hline
\end{tabular}

El enlace de acceso al Story Map es: http://arcg.is/1Rg2PZj

\section{Resultados}

Los resultados obtenidos con esta propuesta metodológica son, en general, muy satisfactorios, ya que se ha logrado aportar un nuevo enfoque metodológico en la enseñanza secundaria de la Geografía -un recurso para el aula- en el que las TIC's juegan un papel fundamental y en el que se pretenden abordar los distintos "temas" o "bloques" que legislativamente se establecen para el estudio de la Geografía, empleando y aprovechando el discurso que ofrecen los distintos paisajes existentes en la superficie terrestre. Consideramos que es un buen recurso para fomentar que la Geografía sea una materia más atractiva e interesante para los estudiantes, y que 
éstos logren desarrollar sus propios conocimientos (capacidad crítica y reflexiva), adaptando así los procesos de enseñanza-aprendizaje a las necesidades e intereses de la sociedad actual, y contribuyendo además, a mostrar el verdadero significado, sentido y utilidad de la Geografía en la interpretación de los procesos recientes, tanto a nivel mundial como regional, relativos a las transformaciones espaciales, los desafíos medioambientales, la geopolítica de los recursos naturales y las fuentes de energía, los procesos de poblamiento y concentración urbana, las desigualdades en el desarrollo humano, o los equilibrios geoestratégicos de fuerzas entre potencias mundiales, por poner algunos ejemplos de los retos a los que se enfrentará la humanidad en las próximas décadas.

Por tanto, la aplicación que hemos desarrollado, y el método que proponemos, tienen la virtualidad de ser desarrollados y adaptados para cualesquiera de los temas tratados en el currículo de Geografía de educación secundaria, proporcionando al profesor y a los alumnos una herramienta de uso relativamente sencillo, que permite incorporar todo tipo de información en los más variados soportes y formatos: esquemas, documentos, audios y vídeos, hiperenlaces, presentaciones, mapas interactivos, ortofotografías aéreas, etc. En las Figuras 3, 4, 5 y 6 incluimos algunas vistas que muestran las posibilidades de la aplicación y que recogen ejemplos diversos de los tipos de recursos y formatos que se pueden incluir. Por último, es interesante señalar que los contenidos también están disponibles para su visualización en dispositivos móviles, lo que sin duda facilita el acceso de los alumnos a los contenidos dado el intenso y frecuente uso que actualmente hacen de los smartphones (Figura 7).

Figura 3.- Vista de la aplicación en la que se muestran ejemplos y esquemas de poblamiento rural.

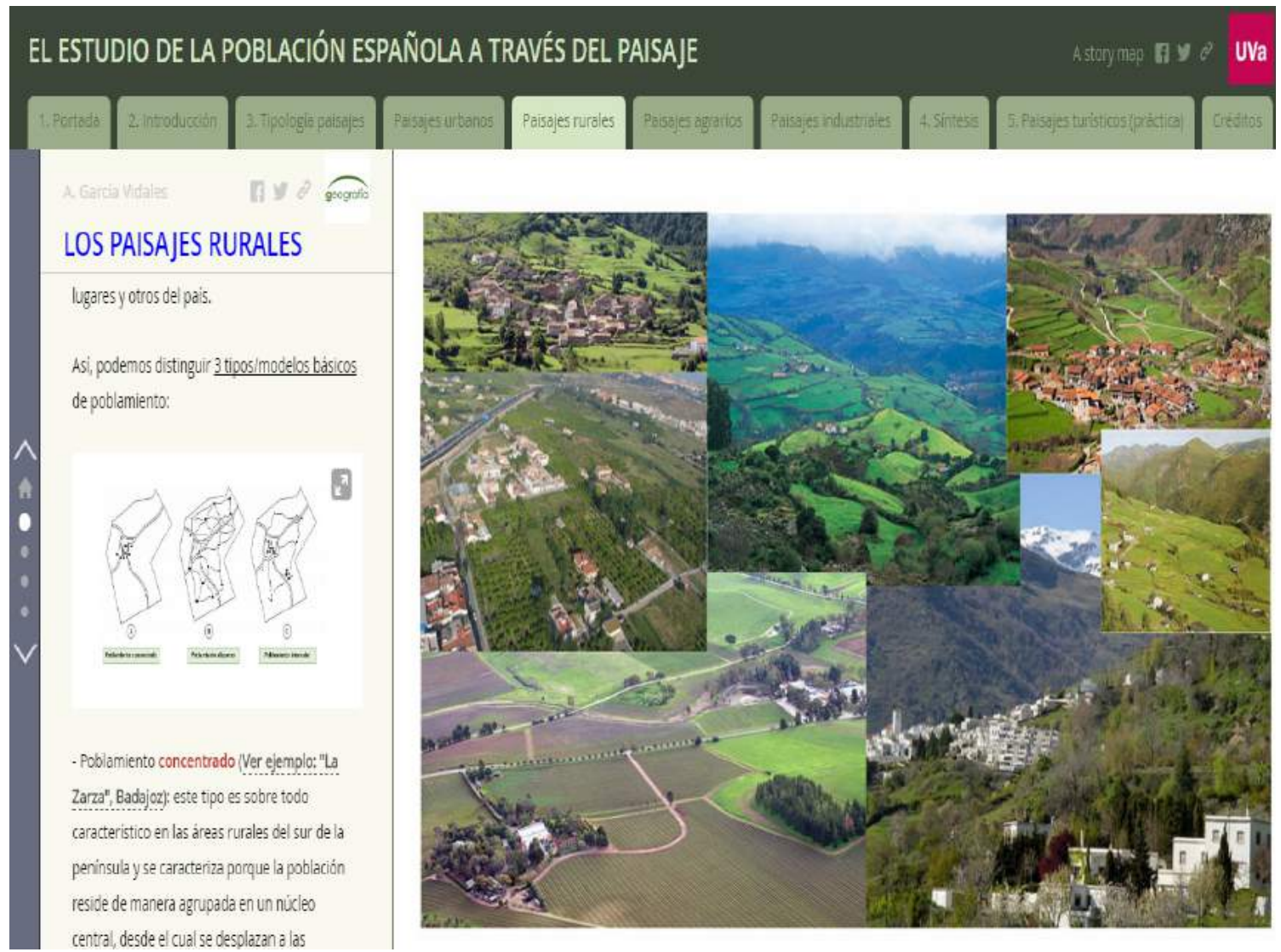


Figura 4.- Vista de la aplicación con mapas interactivos que permiten su comparación y gráficos que ofrecen datos de las regiones.

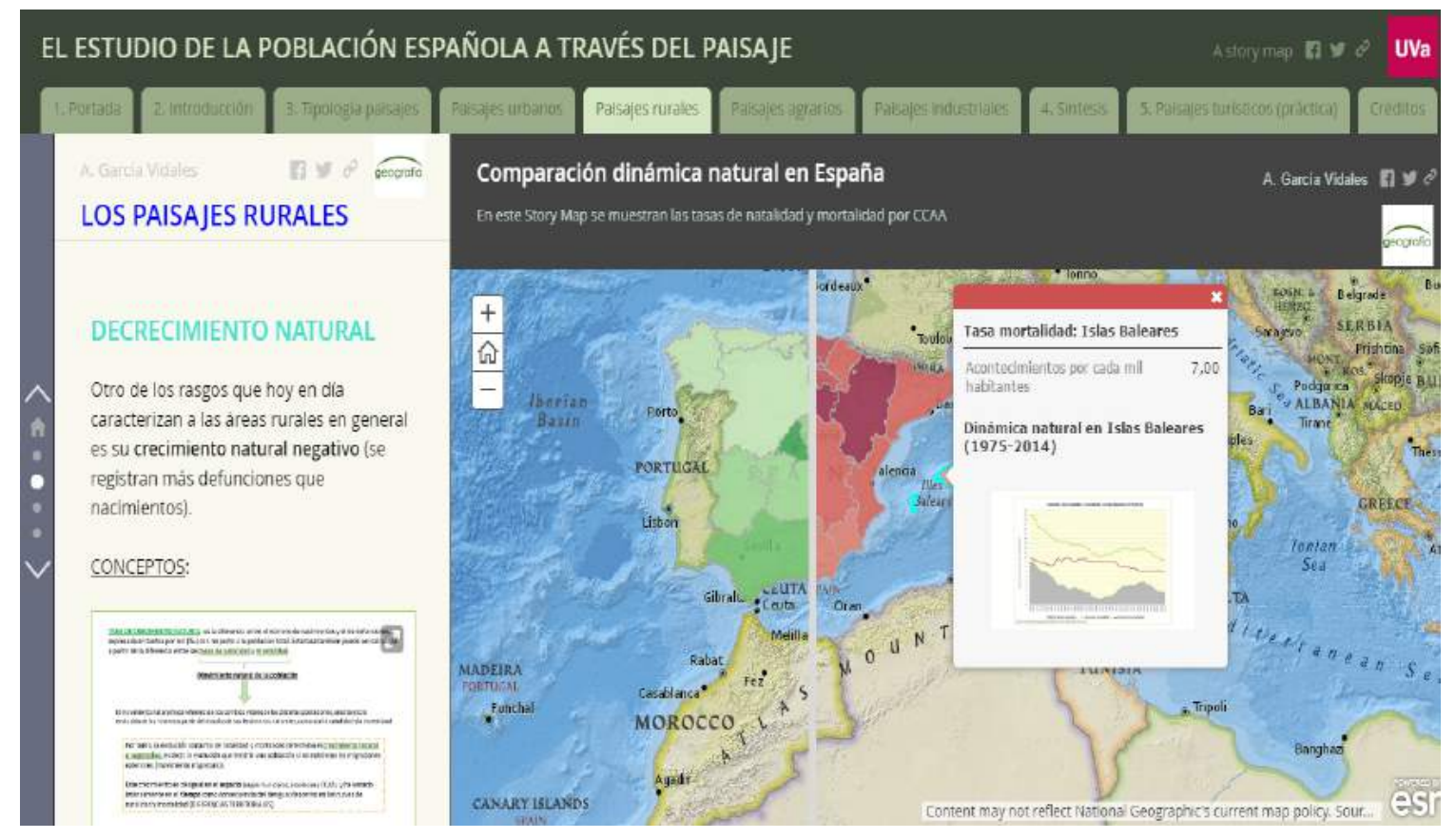

Figura 5.- Vista de la aplicación con ortofotos multitemporales y ejercicios prácticos.

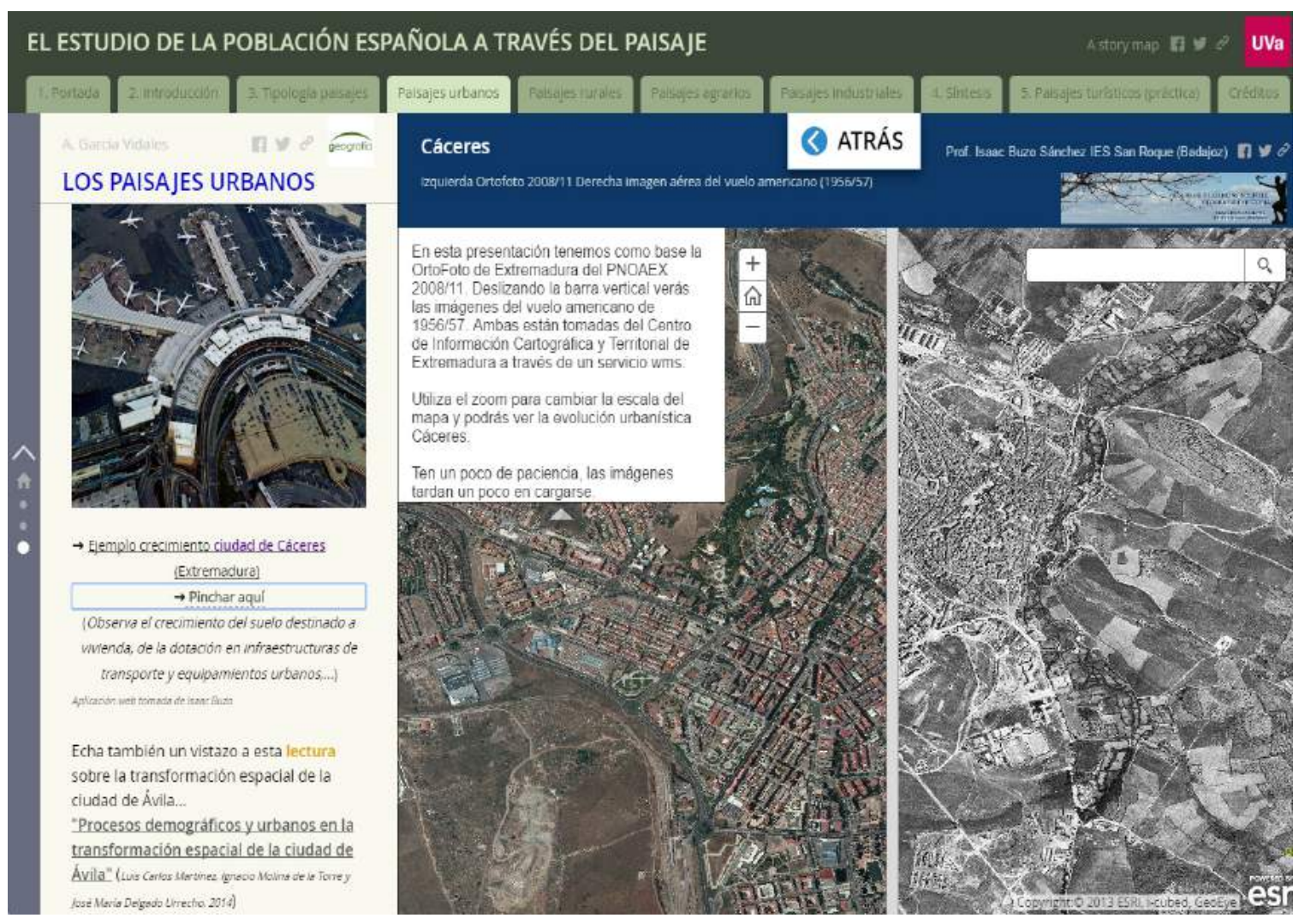


Figura 6.- Vista de la aplicación con vídeos, fotografías y otros recursos para los alumnos.

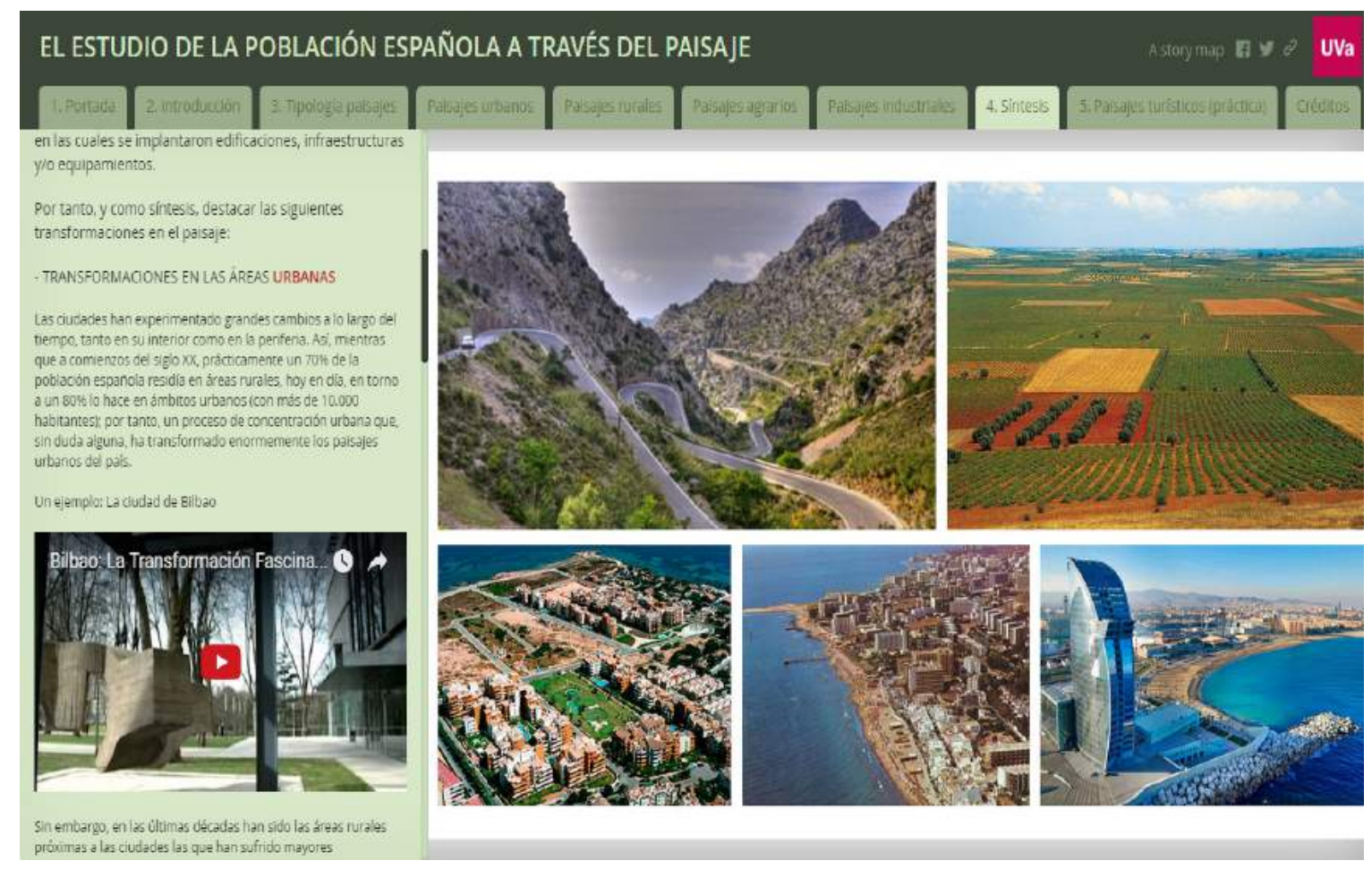

Figura 7.- Ejemplos de la visualización de la Aplicación Web (Story Map) en un dispositivo móvil.
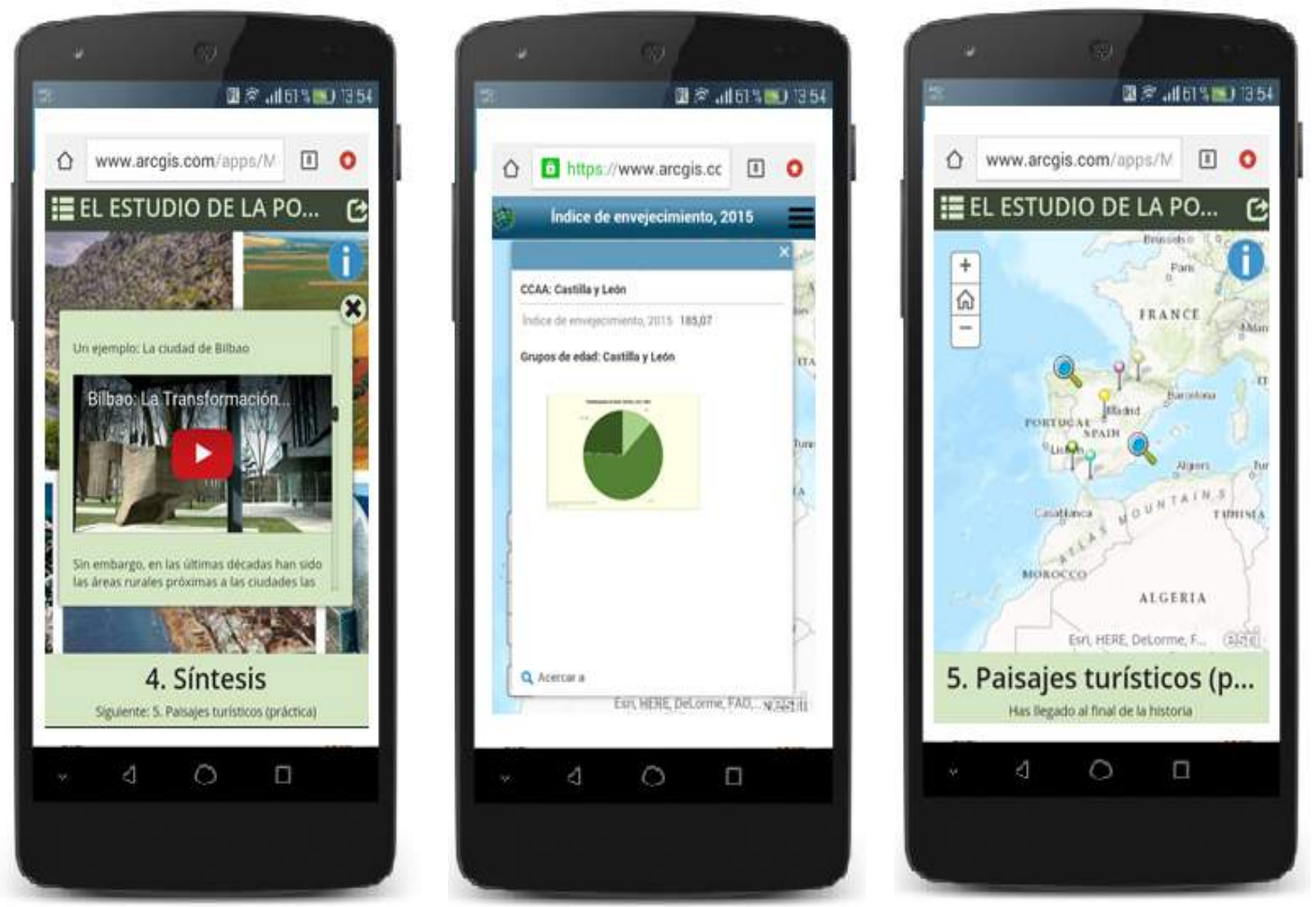
Por todo lo anterior, consideramos que estamos ante un nuevo procedimiento para organizar y ofrecer información a nuestros alumnos, que contribuya a un mejor aprovechamiento de los recursos existentes que se ponen a su disposición, con el fin de aumentar el desarrollo de las tareas de enseñanza-aprendizaje, y al tiempo que se facilita el desarrollo de la cultura digital en el aula. La presentación secuencial de los contenidos, la posibilidad de plasmarlos y localizarlos sobre un mapa, la facilidad para incorporar otros recursos y elementos multimedia, y permitir que los propios alumnos sean sus desarrolladores, hacen que esta herramienta contribuya a un aprendizaje más participativo y colaborativo por parte de los alumnos, abriendo también la posibilidad de encajarlo en un modelo de aprendizaje basado en proyectos (ABP). En todo caso, se trata de una herramienta más que viene a incorporarse a la amplia batería de recursos y propuestas que actualmente están a disposición de los docentes, y serán ellos los que valoren y consideren la conveniencia de su utilización.

\section{Discusión}

Con esta aportación hemos pretendido, por un lado, plantear una nueva forma o metodología didáctica que se adapte mejor a las necesidades tanto formativas como competenciales actuales y, por otro, mostrar el enorme potencial y la utilidad que poseen las TIC como nuevos escenarios de aprendizaje, tal y como ya han puesto de manifiesto otros autores en numerosas ocasiones (Vivancos Martí, 2006; Córcoles, 2010; Saíz Mancha y Picazo Lucas, 2009; Alcántara Manzanares et al., 2014; entre otros). En concreto, y en nuestro trabajo, la herramienta "Story Map" empleada para el estudio de la Geografía en la Enseñanza Secundaria -aunque puede ser perfectamente empleada para otras asignaturas o materias de estudio-, posee una gran variedad de plantillas, con diferentes diseños y recursos que permiten combinar y presentar información en numerosos formatos. Se trata de una aplicación web que es realmente variada y versátil, adaptándose a las necesidades e intereses que, a juicio del docente, sean de mayor importancia en el aprendizaje de los alumnos.

Tal y como señala Álvarez Otero (2014), hoy en día las metodologías de carácter colaborativo con ayuda de las TIC son las que adquieren un papel cada vez más importante, centrando la enseñanza en el aprendizaje del alumno, y en el que las funciones del docente se ajustan y equilibran con las que poseen los discentes. Por tanto, este tipo de metodologías en los procesos de enseñanza-aprendizaje son las que fomentan el aprendizaje que es "aprehendido", aquél que es vivido y forma parte de la experiencia del propio alumno, es decir, forma parte de sus propios conocimientos (Campo País, 2014). De tal forma, y en ese proceso, las TIC y su integración en las aulas juegan un papel esencial, siempre y cuando dicha acción venga acompañada de un cambio -siempre con base didáctica- en la metodología de enseñanza (Cortés Díaz, 2012). La verdadera revolución educativa en las TIC se generará cuando éstas modifiquen los métodos y formas de enseñanza, conformando una parte fundamental del proceso de aprendizaje de los alumnos (Buzo Sánchez, 2014).

En definitiva, un camino de carácter innovador en los procesos de enseñanzaaprendizaje actuales en el que se debe seguir avanzando, ya que tanto la sociedad en su conjunto como los intereses y necesidades de los estudiantes están en constante cambio y evolución, exigiendo así que la educación -y fundamentalmente los docentes- tengan que saber adaptarse correctamente a todo ello, desarrollando nuevos métodos de enseñanza que logren hacer frente a todos estos nuevos retos de la sociedad de la información y la comunicación del siglo XXI (Fajardo Bullón, 2010). 


\section{Referencias}

Alcántara Manzanares, J., Rubio García, S. y Mora Márquez, M. (2014): "Google Earth como herramienta para formadores en la preparación de itinerarios didácticos". En Martínez Medina, R.; Tonda Monllor, E.M. (Eds): Nuevas perspectivas conceptuales y metodológicas para la educación geográfica (AGE), 2, 47-54.

Alcántara Trapero, M.D. (2009): "Importancia de las TIC para la educación". Revista Digital Innovación y Experiencias Educativas, 15, 1-20.

Almerich, G., Suárez, J.M., Belloch, C. y Orellana, N. (2010): "Perfiles del profesorado a partir del conocimiento de los recursos tecnológicos y su relación con el uso que hacen de estas tecnologías". Revista Complutense de Educación, 21(2), 247-269.

Álvarez Otero, J. (2014): "Experiencia colaborativa y TIC en el aula de Geografía: los paisajes turísticos en un mapa de España". En Martínez Medina, R. y Tonda Monllor, E.M. (Eds): Nuevas perspectivas conceptuales y metodológicas para la educación geográfica (AGE), 2, 55-72.

Arrebola Haro, J.C. (2014): "La Tierra de los siete colores: una propuesta interdisciplinar entre ciencias naturales y sociales para la E.S.O. En Martínez Medina, R. y Tonda Monllor, E.M. (Eds): Nuevas perspectivas conceptuales y metodológicas para la educación geográfica (AGE), 2, 73-82.

Bertrand, G. (2008): "Un paisaje más profundo. De la epistemología al método". Cuadernos Geográficos, 43, 17-27.

Bertrand, G. (2010): "Itinerario en torno al paisaje: una epistemología de terreno para tiempos de crisis". Revista Ería, 81, 5-38.

Buzo Sánchez, I. (2014): "Estrategias didácticas innovadoras para la enseñanza de la geografía con una metodología activa". En Martínez Medina, R. y Tonda Monllor, E.M. (Eds): Nuevas perspectivas conceptuales y metodológicas para la educación geográfica (AGE), 2, 11-34.

Campo País, B. (2014): "Estrategias en el ámbito reeducativo para el aprendizaje de la Geografía". En Martínez Medina, R. y Tonda Monllor, E.M. (Eds): Nuevas perspectivas conceptuales y metodológicas para la educación geográfica (AGE), 2, 121138.

Córcoles, J.E. (2010): "Google Earth. Uso didáctico para Escuela 2.0". Revista Digital Sociedad de la Información, 20(3), 1-9.

Cortés Díaz, B. (2012): "Diseño de estrategias didácticas con uso de TIC para el desarrollo de aprendizajes". Revista Educación y Tecnología, 2, 30-40.

De Lázaro y Torres, ML., Izquierdo Álvarez, S. y González González, MJ. (2016): "Geodatos y paisaje: de la nube al aula universitaria". Boletín de la Asociación de Geógrafos Españoles, 70, 371-391.

Delgado Mahecha, O. (1989): "La importancia de la enseñanza de la Geografia". Revista Colombiana de Educación, 20, 101-109.

Fajardo Bullón, F. (2010): "Influencia de las tecnologías de la información y comunicación en la educación". Tejuelo, Monográfico, 4, 9-17.
Gil Duran, N., Calabuig Serra, S. y Medir Huerta, RM. (2014): "El Webmapping como herramienta didáctica para el análisis del paisaje". En Martínez Medina, R. y Tonda Monllor, E.M. (Eds): Nuevas perspectivas conceptuales y metodológicas para la educación geográfica (AGE), 2, 205-218.

Gómez Trigueros, MI. (2010): "Análisis del paisaje físico y humano de la provincia de Alicante: Google Earth como herramienta docente en las clases de Geografia". GeoGraphos, 1(1), 1-26.

González González, MJ. y Pérez Pastrana, A. (2014): "El itinerario didáctico y la técnica del "Storytelling" para desarrollar una metodología activa en Geografía". En Martínez Medina, R. y Tonda Monllor, E.M. (Eds): Nuevas perspectivas conceptuales y metodológicas para la educación geográfica (AGE), 2, 245262.

Herrera Ávila, DL. (2012): "Nuevas tecnologías y educación geográfica: el reto actual". Zona Próxima, 17, 212-223.

Kerski, JJ. (2011): "Sleepwalking into the Future-The Case for Spatial Analysis Throughout Education". En Jekel, T., Koller, A., Donert, K., \& Vogler, R. (Eds.) Learning with GI 2011.

Martín Laborda, R. (2005): "Las nuevas tecnologías en la educación". Cuadernos Sociedad de la Información, 5, 3-34.

Muñoz Carril, PC. y González Sanmamed, M. (2015): “Utilización de las TIC en orientación educativa: Un análisis de las plataformas web en los departamentos de orientación de secundaria". Revista Complutense de Educación, 26(2) 247465.

Oller, M. y Villanueva, M. (2007): "Enseñar Geografía en la educación secundaria: nuevos objetivos, nuevas competencias. Un estudio de caso". Revista Enseñanza de las Ciencias Sociales, 6, 159-168.

Pico, S. (2013): "Formación TIC del profesorado para garantizar el éxito en la integración de la tecnología". Revista Ítaca, 4, 6580.

Rodríguez Eguizabal, AB., y Trabada Crende, XE. (1991): "De la ciudad al campo: el fenómeno social neorruralista en España". Revista Política y Sociedad, 9, 73-86.

Saiz Mancha, A. y Picazo Lucas, C. (2009): Google Earth en el aula. Premio X Certamen de Materiales Curriculares (Castilla La-Mancha). https://t.co/pEzWbSTIMl

Sánchez Asín, A., Boix Peinado, J. y Jurado de los Santos, P. (2009): "La sociedad del conocimiento y las TICs: Una inmejorable oportunidad para el cambio docente". Pixel-Bit, Revista de Medios y Educación, 34, 179-204.

Tello Díaz, J. y Aguaded Gómez, JI. (2009): "Desarrollo profesional docente ante los nuevos retos de las Tecnologías de la Información y la Comunicación en los centros educativos". Pixel-Bit, Revista de Medios y Educación, 34, 3147.

Vivancos, J. (2006): Earthquest y Geoquest: dos propuestas de actividades Geointeractivas. Comunicación: Jornadas sobre Webquest, Barcelona. http://www.xtec.cat/ jvivanco/80minuts/earth\&geoquest_es.pdf 
Libros de texto utilizados para los contenidos de la aplicación Story Map (Educación Secundaria)

Herrero Lorente, J., Cáncer Pomar, L., Fidalgo Hijano, C., Ollero Ojeda, A. y Portugal Ortega, JA. (2000): "Geografía (Bachillerato, Humanidades y Ciencias Sociales)". Editorial Bruño. ISBN: 8421664522 .

Méndez, R., Gutiérrez Puebla, J., Olcina, J. y Pérez-Chacón, E. (2009): "Geografía (20 Bachillerato)". Editorial SM. ISBN: 8467534825 .

Muñoz-Delgado, MC. (2009): "Geografía (20 Bachillerato)". Editorial ANAYA. ISBN: 8466782777.

Legislación en materia de educación:

BOE

- Ley Orgánica 2/2006, de 3 de mayo, de Educación (LOE).

- Ley Orgánica 8/2013, de 9 de diciembre, para la mejora de la calidad educativa (LOMCE).

- Real Decreto 1105/2014, de 26 de diciembre, por el que se establece el currículo básico de la Educación Secundaria Obligatoria y del Bachillerato.

- ORDEN ECD/65/2015, de 21 de enero, por la que se describen las relaciones entre las competencias, los contenidos y los criterios de evaluación de la educación primaria, la educación secundaria obligatoria y el bachillerato.

BOCYL

- ORDEN EDU/1061/2008, de 19 de junio, por la que se regula la implantación y el desarrollo del bachillerato en la Comunidad de Castilla y León.

- ORDEN EDU/362/2015, de 4 de mayo, por la que se establece el currículo y se regula la implantación, evaluación y desarrollo de la educación secundaria obligatoria en la Comunidad de Castilla y León.

- ORDEN EDU/363/2015, de 4 de mayo, por la que se establece el currículo y se regula la implantación, evaluación y desarrollo del bachillerato en la Comunidad de Castilla y León.

La aplicación web en la que se basa este trabajo, se encuentra archivada en WebCite $®$ : http://www.webcitation.org/6nW6vBspq 